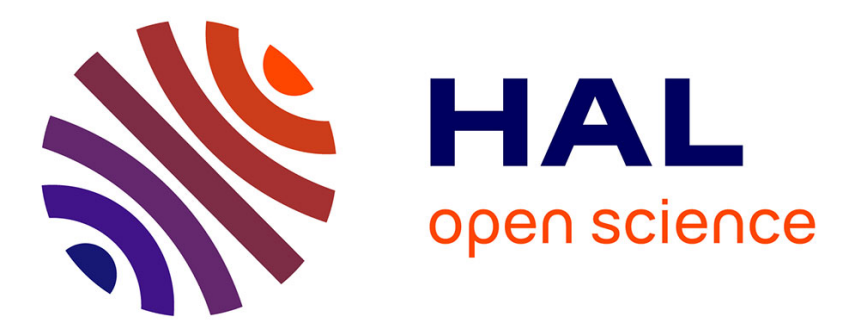

\title{
Evolution of overlaps between configurations in random Boolean networks
}

\author{
B. Derrida, G. Weisbuch
}

\section{To cite this version:}

B. Derrida, G. Weisbuch. Evolution of overlaps between configurations in random Boolean networks. Journal de Physique, 1986, 47 (8), pp.1297-1303. 10.1051/jphys:019860047080129700 . jpa-00210321

\section{HAL Id: jpa-00210321 https://hal.science/jpa-00210321}

Submitted on 1 Jan 1986

HAL is a multi-disciplinary open access archive for the deposit and dissemination of scientific research documents, whether they are published or not. The documents may come from teaching and research institutions in France or abroad, or from public or private research centers.
L'archive ouverte pluridisciplinaire HAL, est destinée au dépôt et à la diffusion de documents scientifiques de niveau recherche, publiés ou non, émanant des établissements d'enseignement et de recherche français ou étrangers, des laboratoires publics ou privés. 


\title{
Evolution of overlaps between configurations in random Boolean networks
}

\author{
B. Derrida \\ Service de Physique Théorique, CEN Saclay, F-91191 Gif-sur-Yvette Cedex, France \\ and $\mathrm{G}$. Weisbuch \\ Groupe de Physique du Solide de l'E.N.S. 24 rue Lhomond, F-75231 Paris Cedex 5, France
}

(Reçu le 6 mars 1986, accepté le 23 avril 1986)

\begin{abstract}
Résumé. - Les réseaux booléens aléatoires sont constitués d'unités logiques binaires connectées aléatoirement. A chaque intervalle de temps $t$, chaque unité, ou spin, prend la valeur 0 ou 1 suivant une fonction booléenne de $K$ signaux d'entrée binaires provenant des $K$ spins connectés. Nous comparons l'évolution au cours du temps des recouvrements entre des configurations initialement différentes pour les deux modèles suivants : dans le modèle de Kauffman, les connexions et les fonctions booléennes des automates sont choisies une fois pour toutes à l'instant initial. Dans le modèle recuit. ces paramètres font l'objet d'un nouveau tirage aléatoire à chaque pas de temps. Les simulations numériques effectuées pour les deux modèles sont dans un accord remarquable avec les prédictions théoriques faites pour le second modèle.
\end{abstract}

\begin{abstract}
Random Boolean nets are systems of randomly connected binary units (or spins). Each spin $\sigma_{i}$ can take two possible values $\left(\sigma_{i}=0\right.$ or 1$)$. It receives, at time $t, K$ binary input signals coming from $K$ connected spins and updates its state according to a deterministic Boolean function of the $K$ inputs. We compare the time evolution of the overlaps between different configurations for the two following models : Kauffman's model, for which the connections and Boolean function of each spin are randomly chosen at time $t=0$ and remain unchanged at later times; the annealed model, for which these parameters are randomly reset at each time step. The numerical simulations for both models agree remarkably well with the theoretical predictions available for the second model.
\end{abstract}

\section{Introduction.}

Random Boolean nets were proposed by Kauffman [1] as models of genetic systems. They are also used as models for disordered systems when one is interested by dynamical properties rather than by static equilibrium properties. As compared to formal neurons nets, such as proposed by Hopfield [2], they differ by the fact that an energy function is not available since interactions among automata are not symmetric. This is the reason why attractors are more complex : there can be limit cycles with eventually large periods. The absence of an energy function also complicates the theory and the prediction of the dynamical properties. Even the simple scaling laws observed by Kauffman [1] from computer simulations have not yet been formally established [3-6]. Recently another approach to this fifteen year old problem has been proposed [7], which allows theoretical predictions about a closely related model (the annealed model). This approach allows the analytic calculation of the time evolution of the distances between pairs of configurations in the annealed model.

In the present work, we first generalize the analytic calculations on the annealed model to the time evolution of triplets, quartets and quintets of configurations. We then compare these analytical predictions for the annealed model with numerical simulations done for the quenched model.

Our main results are the following :

1 . When one iterates $n$ configurations $(n \geqslant 2)$ for the annealed model, the number $x_{2}$ of spins which are identical in 2 configurations has a limit at infinite time, which is the same for all pairs [7]. Similarly, the number $x_{i}$ of spins which are identical in $i$ configurations has a limit which is independent of the $i$-plet. If one knows 
the $x_{i}$ for even $i\left(x_{2}, x_{4}, \ldots, x_{2 p}\right)$, the values of $x_{i}$ for odd $i\left(x_{3}, x_{5}, \ldots, x_{2 p+1}\right)$ can be deduced. Wę shall write the equations giving $x_{2}, x_{3}, x_{4}$ and $x_{5}$.

2. In the thermodynamic limit (i.e. when the number $N$ of automata becomes infinite), one cannot distinguish the numerical simulations done for the quenched model from the analytic predictions done for the annealed model.

\section{Definitions.}

Let us briefly recall the definitions of the quenched model (Kauffman's model) and of the annealed model.

2.1 THE QUENCHED MODEL. - The quenched model depends on a parameter $K$. The system consists of a net of $N$ spins $\sigma_{i}$ which can take 2 possible values $\left(\sigma_{i}=0\right.$ or 1$)$. The time evolution of this system is given by $\mathrm{N}$ Boolean functions of $K$ variables each.

$$
\sigma_{i}^{(t+1)}=f_{i}\left(\sigma_{i_{1}}^{(t)}, \sigma_{i_{2}}^{(t)}, \ldots, \sigma_{i_{K}}^{(t)}\right)
$$

For each $i$ the spins $\sigma_{i_{1}}, \sigma_{i_{2}}, \ldots, \sigma_{i_{K}}$ are randomly chosen among the $N$ spins $\left(\sigma_{i_{1}}, \sigma_{i_{2}}, \ldots, \sigma_{i_{K}}\right.$ are the inputs of spin $\sigma_{i}$ ). The system is defined once a function $f_{i}$ and the input sites $i_{1}, i_{2}, \ldots, i_{K}$ have been chosen for each site $i$ of the net. At each time step (from $t$ to $t+1$ ), all the spins are simultaneously updated according to (1).

There exists $2^{2^{K}}$ possible Boolean functions $f_{i}$ of $K$ variables. In Kauffman's model each function $f_{i}$ is randomly chosen among these $2^{2^{K}}$ possible functions. The system is random because for each $i$ the input sites $i_{1}, i_{2}, \ldots, i_{K}$ and the function $f_{i}$ are randomly chosen. This randomness is quenched because the input sites $i_{1}, i_{2}, \ldots, i_{K}$ and the function $f_{i}$ are quenched : they remain constant over time.

The dynamics of such a net is thus fully deterministic. Therefore, since the system has only $2^{N}$ different configurations, after a time $t>2^{N}$, the system must have been at least twice in the same configuration, and so the system must be periodic with a period $P$ less or equal to $2^{N}$.

The dynamical properties of the quenched model were studied by numerical simulations $[1,5]$ Kauffman [1] reports that the period $P$ of the limit cycles follows different regimes depending on $K$. For low $K(K=1$ or 2$), P$ increases as the square root of the number $N$ of automata. For $K \geqslant 3, P$ increases exponentially with $N$. Numerous attempts [1,3-6] to derive these scaling laws were made without achieving a complete success, except for $K=N$ [8].

2.2 THE ANNEALED MODEL. - The definition of the annealed model is exactly the same as the one of the quenched model. In particular the connectivity constant $K$ plays the same role. The only difference with the quenched model is that the connection structure (the inputs $i_{1}, i_{2}, \ldots, i_{K}$ of each site $i$ ) and the Boolean function $f_{i}$ are randomly changed at each time step. Annealed nets are thus nets of probabilistic automata. Therefore they cannot show any periodic behaviour.

So the two models look a priori very different since the quenched model has limit cycles which are absent in the annealed model.

We shall see however that one can compare the time evolution of overlaps between configurations in the two models and that in the thermodynamic limit $(N \rightarrow \infty)$, the two models have identical behaviours as far as overlaps are concerned.

\section{Overlaps between configurations.}

In the next section (section 4), we are going to study the time evolution of the overlaps between configurations in the annealed model. Before doing so, we want to discuss in the present section the following question. Given $n$ configurations $\mathrm{C}_{1}, \mathrm{C}_{2}, \ldots, \mathrm{C}_{n}$ of the spins $\sigma_{i}$, i.e. given $\sigma_{i}^{\mu}$ for $1 \leqslant i \leqslant N$ for each configurations $\mathrm{C}_{\mu}$, how many parameters are needed in order to describe all the overlaps between these configurations?

Let us denote by $A_{\mu \nu}$ the number of spins $\sigma_{i}$ which are the same in $\mathrm{C}_{\mu}$ and $\mathrm{C}_{v}$, i.e. the number of spins $\sigma_{i}$ such that $\sigma_{i}^{\mu}=\sigma_{i}^{\nu}$. Similarly, one can define $A_{\lambda \mu \nu}$ as the number of spins which are identical in $\mathrm{C}_{\lambda}, \mathrm{C}_{\mu}$ and $\mathrm{C}_{v}$. And so on. So for example if we have $n=8$ configurations, $A_{13567}$ is the number of spins $\sigma_{i}$ such that $\sigma^{1}=\sigma^{3}=\sigma^{5}=\sigma^{6}=\sigma^{7}\left(\sigma^{2}, \sigma^{4}, \sigma^{8}\right.$ being anything).

When the number $N$ of spins is large, one can define intensive quantities $a_{\lambda \mu \nu \ldots}$ by

$$
a_{\lambda \mu \nu \ldots}=A_{\lambda \mu \nu \ldots} / N \text {. }
$$

$A$ priori, for a set of $n$ configurations, there are $2^{n}-n-1$ such quantities $a_{\lambda \mu \nu \ldots .}$. The question one can ask is the following. Are they independent or are there relations between them ?

It turns out that for Ising $\operatorname{spins}\left(\sigma_{i}=0,1\right.$ or $\left.\sigma_{i}= \pm 1\right)$, only the even ones are independent and all the odd ones can be expressed as a function of the even ones (even or odd refer to the number of indices of the quantity $\left.a_{\lambda \mu \nu . . .}\right)$. For example, we will prove that

$$
\begin{gathered}
a_{123}=\left(a_{12}+a_{13}+a_{23}-1\right) / 2 \\
a_{12345}=1-\left(a_{12}+a_{13}+a_{14}+a_{15}+a_{23}+\right. \\
\left.+a_{24}+a_{25}+a_{34}+a_{35}+a_{45}\right) / 4 \\
+\left(a_{1234}+a_{1235}+a_{1245}+a_{1345}+a_{2345}\right) / 2
\end{gathered}
$$

One can prove that for an arbitrary number $n$ of configurations, odd $a$ 's are always functions of even $a$ 's. One could also try to generalize formulae (3) and (4). But since in the rest of the paper we will never consider sets of more than 5 configurations, we will not discuss here the problem of general $n$ because this would be too long and would require the introduction of heavy notations. So we will limit ourselves to the proof of (3) and (4). 
Let us first prove (3). If we have 3 configurations $\mathrm{C}_{1}$, $\mathrm{C}_{2}, \mathrm{C}_{3}$, let us call $N x_{v}$ the number of spins $\sigma$ such that $\sigma^{\lambda}=\sigma^{\mu}=1-\sigma^{\nu}$. Then one has

$$
\begin{aligned}
& a_{12}=a_{123}+x_{3} \\
& a_{13}=a_{123}+x_{2} \\
& a_{23}=a_{123}+x_{1} \\
& 1=a_{123}+x_{1}+x_{2}+x_{3} .
\end{aligned}
$$

This last equation expresses just the fact that a spin is either the same in the 3 configurations or differs in one configuration from the two others. Eliminating the $x_{v}$, one gets equation (3).

Similarly, to prove equation (4), one can define $N y_{5}$ as the number of spins $\sigma_{i}$ such that $\sigma_{i}^{1}=\sigma_{i}^{2}=\sigma_{i}^{3}=$ $\sigma_{i}^{4}=1-\sigma_{i}^{5}$ and $H z_{45}$ the number of spins $\sigma_{i}$ such that $\sigma_{i}^{1}=\sigma_{i}^{2}=\sigma_{i}^{3}=1-\sigma_{i}^{4}=1-\sigma_{i}^{5}$ (of course $y_{\mu}$ and $z_{\lambda \mu}$ are defined in a similar way for all $\lambda$ and $\mu$ ). Then one has

$$
\begin{aligned}
a_{1234} & =a_{12345}+y_{5} \\
a_{123}= & a_{12345}+y_{4}+y_{5}+z_{45} \\
a_{12}= & a_{12345}+y_{5}+y_{4}+y_{3}+z_{45}+z_{35}+ \\
&
\end{aligned}
$$

Plus all permutations of these first 3 equations and

$$
1=a_{12345}+\sum_{\mu=1}^{5} y_{\mu}+\sum_{\mu=1}^{4} \sum_{\nu=\mu+1}^{5} z_{\mu \nu} .
$$

This last equation expresses again that the total number of spins is $N$. Eliminating again the $y$ 's and $z$ 's, one gets equation (4).

At the end of this section, we can make two remarks.

- If the spins were not Ising spins but Potts spins $\left(\sigma_{i}=1,2,3, \ldots, q\right)$ i.e. would have $q$ possible values instead of 2 in the Ising case, then one could not relate the odd $a$ 's to the even $a$ 's.

- One can wonder whether all the even $a$ 's are independent or not. Let us just give a simple example which shows that the $a$ with 4 indices cannot be expressed as a function of the $a$ 's with 2 indices. Consider the two following sets of 4 configurations 4 spins.

$$
\begin{array}{cc}
\mathrm{C}_{1}=1111 & \mathrm{C}_{1}=1111 \\
\mathrm{C}_{2}=1100 & \mathrm{C}_{2}=1100 \\
\mathrm{C}_{3}=1010 & \mathrm{C}_{3}=1010 \\
\mathrm{C}_{4}=1001 & \mathrm{C}_{4}=0110 \\
\text { Case I } & \text { Case II }
\end{array}
$$

In both cases $A_{\mu \nu}=2, \forall \mu$ and $v$, but $A_{1234}=1$ in case I and $A_{1234}=0$ in case II. So clearly $A_{1234}$ cannot be expressed as a function of the $A_{\mu v}$.

\section{Time evolution of the overlaps in the annealed model.}

In this section, we are going to generalize the calculation of the time evolution of the distance between pairs of configurations (Derrida and Pomeau, 1986)

[7] to the case of the overlap of $n$ configurations.

We keep the notations of the previous section. Let $N a_{\lambda \mu \nu . .}$ be the number of spins which are the same in configurations $\mathrm{C}_{\lambda}, \mathrm{C}_{\mu}, \mathrm{C}_{\boldsymbol{v}} \ldots$ at time $t$. Let us define by $N a_{\lambda \mu \nu \ldots}^{\prime}$ the number of spins which are the same in configurations $\mathrm{C}_{\lambda}, \mathrm{C}_{\mu}, \mathrm{C}_{v} \ldots$ at time $t+1$. In reference [7], it was shown that the time evolution of $a_{\lambda v}$ was given by :

$$
a_{12}^{\prime}=\left[1+\left(a_{12}\right)^{K}\right] / 2 .
$$

Let us recall briefly how one can get (7). Among the $N$ spins, $N\left(a_{12}\right)^{K}$ have all their inputs the same in $\mathrm{C}_{\mu}$ and $C_{v}$ and $N\left[1-\left(a_{12}\right)^{K}\right]$ have at least one of their inputs different. The spins which have all their inputs the same in $C_{1}$ and $C_{2}$ will be the same in the two configurations at time $t+1$. On the contrary, the spins which have at least one of their inputs different at time $t$, have a probability $1 / 2$ of being the same at time $t+1$ and $1 / 2$ of being different at time $t+1$. Therefore

$$
a_{12}^{\prime}=a_{12}^{K}+\left[1-\left(a_{12}\right)^{K}\right] / 2
$$

which gives (7).

Let us now discuss the case of 3 configurations. The time evolution of $a_{12}, a_{13}$ and $a_{23}$ will be given by (7). Let us now calculate $a_{123}^{\prime}$. One has

$$
\begin{aligned}
a_{123}^{\prime}= & a_{123}^{K}+ \\
& +\left(a_{12}^{K}-a_{123}^{K}\right) / 2+\left(a_{13}^{K}-a_{123}^{K}\right) / 2+\left(a_{23}^{K}-a_{123}^{K}\right) / 2 \\
& +\left(1-a_{12}^{K}-a_{13}^{K}-a_{23}^{K}+2 a_{123}^{K}\right) / 4
\end{aligned}
$$

In (9), the first term represents the spins which have all their inputs the same in $C_{1}, C_{2}$ and $C_{3}$. The second term represents the spins which have all their inputs the same in $C_{1}$ and $C_{2}$ but at least one input differs in $\mathrm{C}_{3}$. These spins have a probability $1 / 2$ of being the same in $\mathrm{C}_{1}, \mathrm{C}_{2}$ and $\mathrm{C}_{3}$ at time $t+1$. The similar terms for the pairs $\left(\mathrm{C}_{1}, \mathrm{C}_{3}\right)$ and $\left(\mathrm{C}_{2}, \mathrm{C}_{3}\right)$ are the next two terms. Lastly, the last term represents the spins which have all their inputs the same in none of the pairs $\left(C_{1}, C_{2}\right),\left(C_{2}, C_{3}\right)$ or $\left(C_{1}, C_{3}\right)$. These spins have a probability $1 / 4$ of being the same in the 3 configuratons at time $t+1$. One can simplify (9) and one gets

$$
a_{123}^{\prime}=\left(a_{12}^{K}+a_{13}^{K}+a_{23}^{K}+1\right) / 4 .
$$

If one uses (7) to express the $a_{\mu \nu}$ in terms of the $a_{\mu \nu}^{\prime}$ one gets :

$$
a_{123}^{\prime}=\left(a_{12}^{\prime}+a_{13}^{\prime}+a_{23}^{\prime}-1\right) / 2
$$

which is again the relation (3) found in section 3. So the time evolution of $a_{123}$ could have been guessed directly from (3) without any calculations.

One can use the same procedure and get the time evolution $a_{1234}$. The reasoning is exactly the same although it becomes longer and more complicated. 
So we will just give the answer

$$
\begin{aligned}
a_{1234}^{\prime} & =1 / 8+\left(a_{12}^{K}+a_{13}^{K}+a_{14}^{K}+a_{23}^{K}+a_{24}^{K}+a_{34}^{K}\right) / 8+\left[\left(2-a_{13}-a_{14}-a_{23}-a_{24}+4 a_{1234}\right) / 2\right]^{K} / 8 \\
& +\left[\left(2-a_{12}-a_{14}-a_{23}-a_{34}+4 a_{1234}\right) / 2\right]^{K} / 8+\left[\left(2-a_{12}-a_{13}-a_{24}-a_{34}+4 a_{1234}\right) / 2\right]^{K} / 8 \\
& -2\left(a_{1234}\right)^{K} / 8 .
\end{aligned}
$$

In principle, one can carry on and compute the overlaps for a larger number of configurations but the calculations become more and more complicated and we did not find yet the expression in the general case.

Obviously, one does not need to do the calculation for odd $a$ 's because of what was said in section 3 and because the time evolution of $a_{12345}$ can be obtained from (7) and (12) using equation (4).

\section{Results of computer simulations of the quenched and of the annealed models.}

In this section, we are going to present the time evolution of the $a_{\lambda \mu \nu . . .}$ obtained by numerical simulations for both the quenched and the annealed models.

Our procedure is the following. For a net of $N$ spins $(N=32,256,2048$ and 16384$)$, we choose at random at time $t=0,4$ configurations $\mathrm{C}_{1}, \mathrm{C}_{2}, \mathrm{C}_{3}, \mathrm{C}_{4}$. Each spin $\sigma_{i}$ in each configuration is chosen to be 1 with probability $1 / 2$ and 0 with probability $1 / 2$. Then, we build the net (i.e. for each spin $\sigma_{i}$ we choose its $K$ inputs $i_{i}, \ldots, i_{K}$ and the function $f_{i}$ at random). Then we compute the configurations $\mathrm{C}_{1}, \mathrm{C}_{2}, \mathrm{C}_{3}$ and $\mathrm{C}_{4}$ at time $t=1$. For the quenched model, we keep the $i_{i}, \ldots, i_{\mathrm{K}}$ and the $f_{i}$ fixed. Whereas we change them for the annealed model. Then we compute the 4 configurations at time $t=2$. Again, we change the rules for the annealed model and we keep them fixed for the quenched model. And so on. Doing that, we can measure $a_{12}(t), a_{123}(t), a_{1234}(t)$ for both the quenched and the annealed models. So we get the time evolution $0<t \leqslant 50$ of these 3 quantities.

For small sizes $(N=32,256)$, the sample to sample fluctuations are rather large. So we decided to average these quantities over many samples. We averaged over 50 samples for $N=16384,400$ samples for $N=2048$, 3200 samples for $N=256,25600$ samples for $N=32$, so that the amount of computer time devoted to each size is roughly the same.

In the figures 1 and 2 , we plot the averaged values of $a_{12}(t)$ and $a_{1234}(t)$ versus time for sizes $N=32$, 256, 2048 and 16384 for nets with $K=3$. We do not show $a_{123}(t)$ because the curves are very similar.

The full curve represents the result obtained from expressions (10) and (12). Since at time $t=0$, the 4 configurations are chosen at random, one has on average :

$$
\begin{aligned}
a_{12}= & a_{13}=a_{14}=a_{23}=a_{24}=a_{34}=1 / 2 \\
& a_{123}=a_{124}=a_{134}=a_{234}=1 / 4 \\
& a_{1234}=1 / 8
\end{aligned}
$$

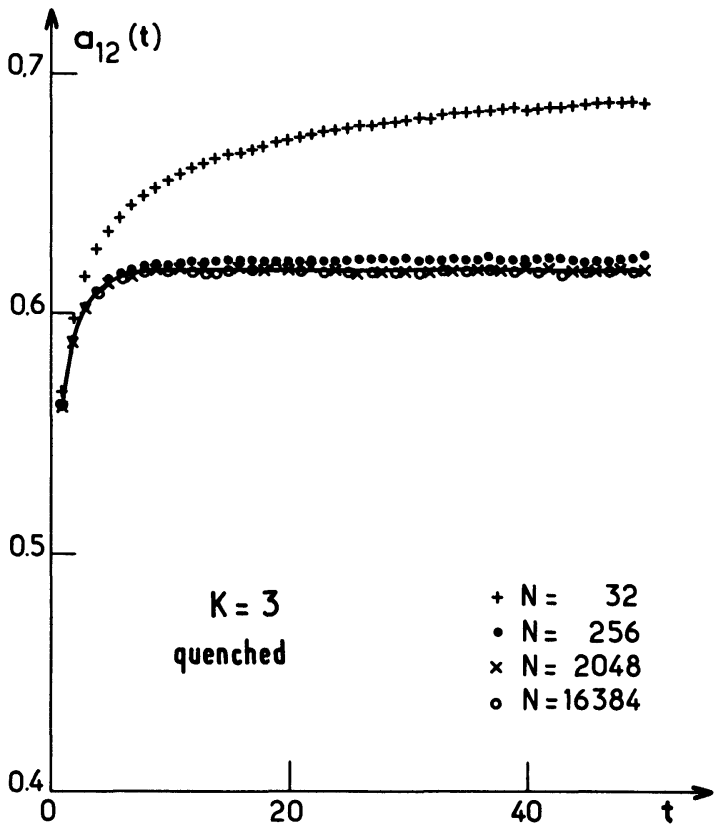

a)

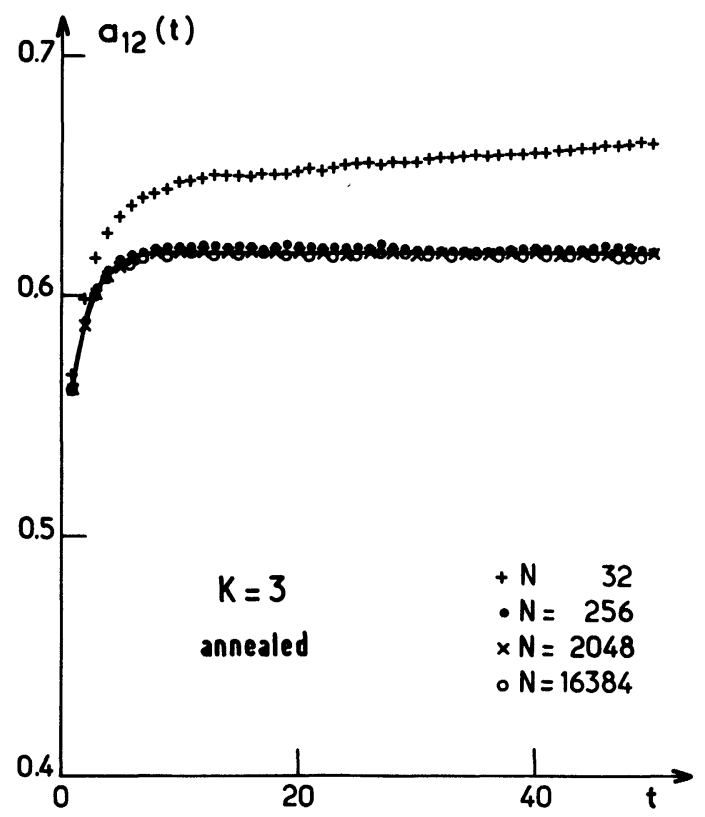

b)

Fig. 1. - Time evolution of the overlaps between 2 randomly chosen configurations for several sizes of a random Boolean network of connectivity $K=3$. $[N=32(+)$, $N=256(0), N=2048(\times)$ and $N=16384(0)]$. The full curve represents $x_{2}(t)$, the result of expression (15). Figure 1a gives the simulation results of the quenched model and figure $1 \mathrm{~b}$ the simulation results of the annealed model. 


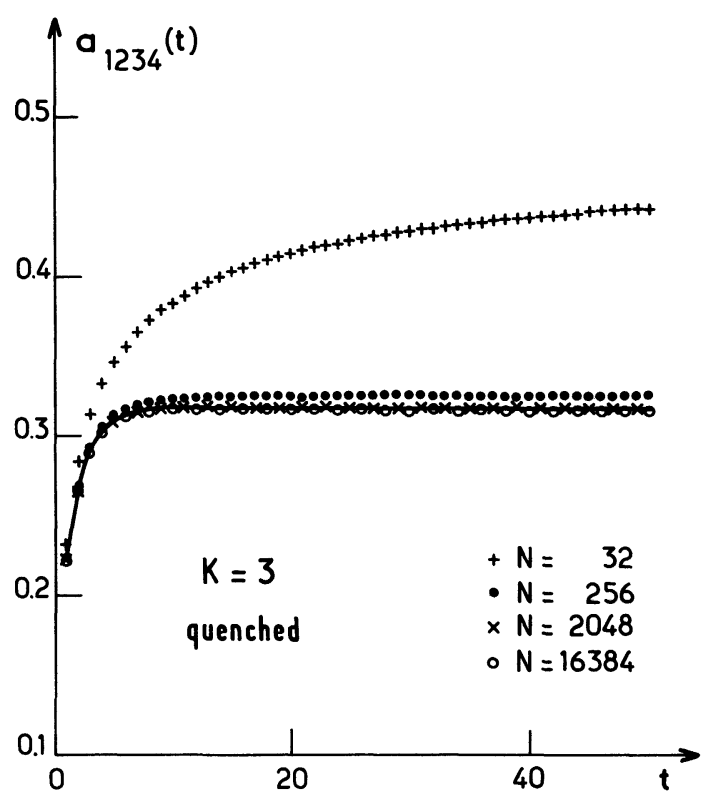

a)

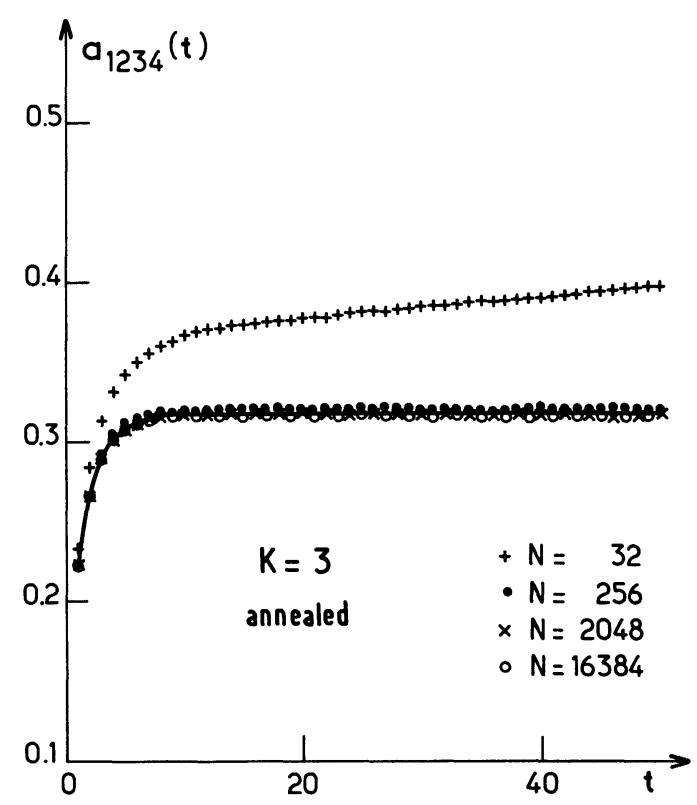

b)

Fig. 2. - Same conditions as in figure 1, except that we have plotted the overlaps between 4 configurations $a_{1234}(t)$, and that the full curve corresponds to $x_{4}(t)$ given by expression (16).

Since all the $a_{\mu v}$ are the same at time $t=0$, they will remain equal at later times. Therefore if one defines $x_{2}(t), x_{3}(t)$, and $x_{4}(t)$ by

$$
\begin{aligned}
& x_{2}(t)=a_{12}(t)=a_{13}(t)=\cdots \\
& x_{3}(t)=a_{123}(t)=a_{124}(t)=\cdots \\
& x_{4}(t)=a_{1234}(t)
\end{aligned}
$$

then the time evolution of $x_{2}(t)$ and $x_{4}(t)$ are given from (12) and (13) by

$$
\begin{gathered}
x_{2}(t+1)=\left[1+\left(x_{2}(t)\right)^{K}\right] / 2 \\
x_{4}(t+1)=1 / 8+3\left[x_{2}(t)\right]^{K} / 4+ \\
+3\left[1-2 x_{2}(t)+2 x_{4}(t)\right]^{K} / 8-\left[x_{4}(t)\right]^{K} / 4
\end{gathered}
$$

with the initial values

$$
x_{2}(0)=1 / 2 \text { and } x_{4}(0)=1 / 8 .
$$

So $x_{2}(t)$ and $x_{4}(t)$ are the predictions of the calculations done in section 4 . The full line represents $x_{2}(t)$ in figure 1 and $x_{4}(t)$ in figure 2.

It is easy to see from (3) and (4) that

$$
\begin{aligned}
& x_{3}(t)=\left[3 x_{2}(t)-1\right] / 2 \\
& x_{5}(t)=1-5 x_{2}(t) / 2+5 x_{4}(t) / 2 .
\end{aligned}
$$

In figures 3 and 4, we represent the same quantities as in figures 1 and 2 in the case $K=2$.

Again as $N$ increases, one sees that the results become closer and closer to the theoretical predictions $x_{2}(t)$ and $x_{4}(t)$ given by expressions $(15,16,17)$. So again within the accuracy of our calculation, we see that as $N \rightarrow \infty$, the results for the quenched and for the annealed models are the same.

For $K=2$, we netherless see that the convergence (as $N$ increases) is slower than for $K=3$.

The formulae of section 4 and therefore the predictions given by (15), (16) and (17) are valid in principle for the annealed model in the thermodynamic limit $(N \rightarrow \infty)$. For finite $N$, even the data for the annealed model exhibit some finite size effects (see Figs. 1b, 2b, $3 b, 4 b)$.

It is interesting to have an idea of the amplitude of these finite size effects. For the quenched model, they are stronger for $K=2$ than for $K=3$.

Let us restrict our analysis of finite size effects to the quenched model for $K=2$. We see in figures $3 a$ and $4 \mathrm{a}$ that, as $t \rightarrow \infty, x_{2}(t)$ and $x_{4}(t)$ computed from formulae (15) and (16) converge to 1 . Whereas for finite $N$, the averaged values of $a_{12}(t)$ and $a_{1234}(t)$ saturate as $t \rightarrow \infty$. Let us denoye by $b_{12}$ and $b_{1234}$ their limits as $t \rightarrow \infty$

$$
\begin{aligned}
& a_{12}(t) \rightarrow b_{12} \\
& a_{1234}(t) \rightarrow b_{1234}
\end{aligned}
$$

In figure 5 , we have plotted $\log \left(1-b_{12}\right)$ and $\log (1-$ $\left.b_{1234}\right)$ versus $\log N$. We have estimated $b_{12}$ and $b_{1234}$ by computing $a_{12}(t)$ and $a_{1234}(t)$ at time $t=50$ and $t=100$ to make sure that the limits had been reached. In figure 5 , we show the results for $N=8,16,32 \ldots 512$. For larger sizes $N$, the values at time $t=50$ and $t=100$ had not yet reached saturation and we did not get reliable values of $b_{12}$ and $b_{1234}$. 


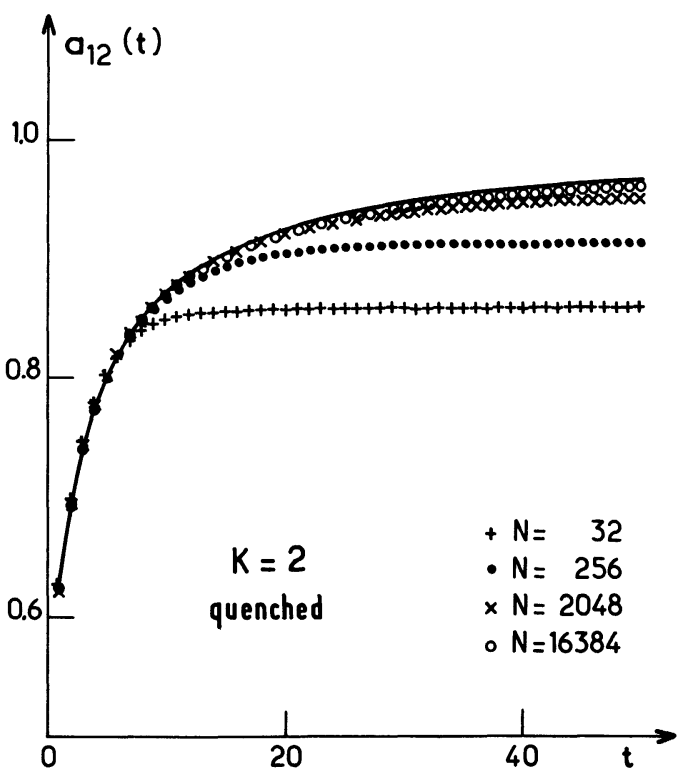

a)

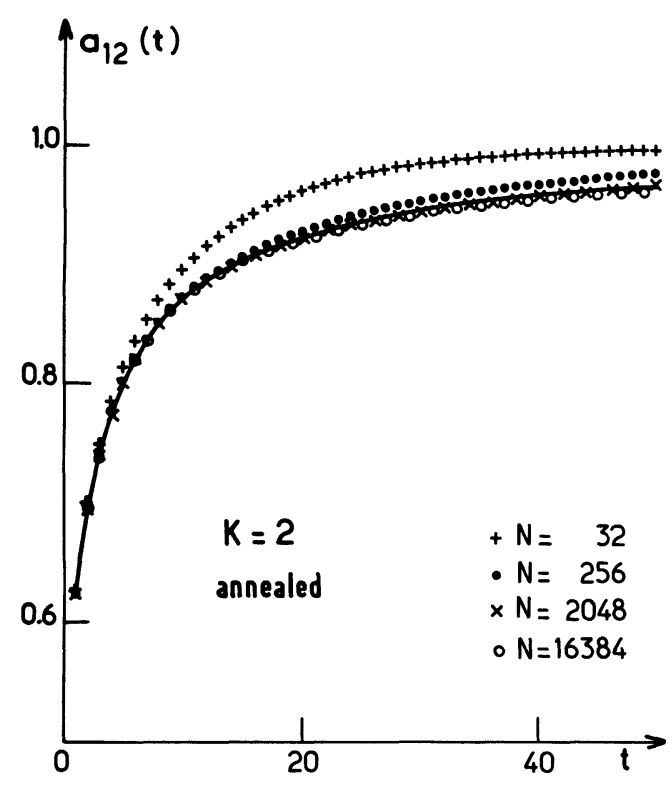

b)

Fig. 3. - Same conditions as in figure 1 for connectivity $K=2$ (overlaps between 2 configurations).

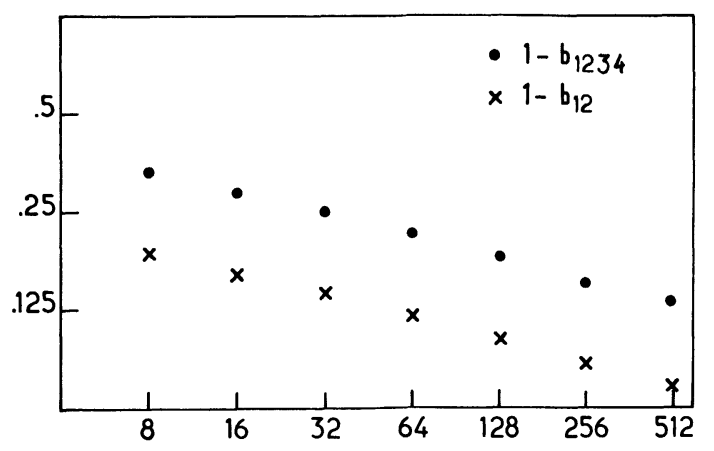

Fig. 5. - Finite size effects in the quenched model for $K=2$. Log-Log plot of $1-b_{12}$ and $1-b_{1234}$, defined by equations (20) and (21) versus $N$.

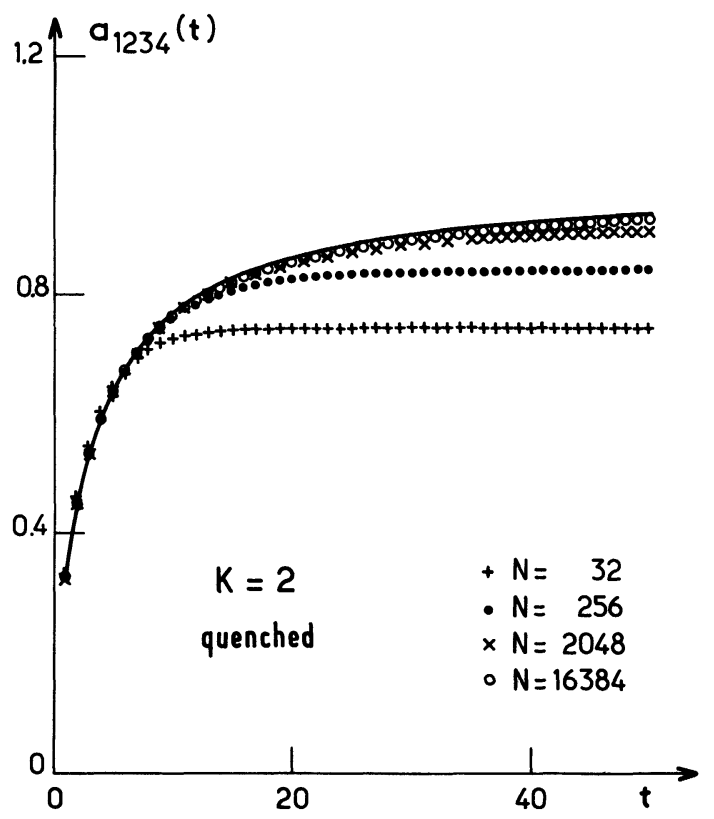

a)

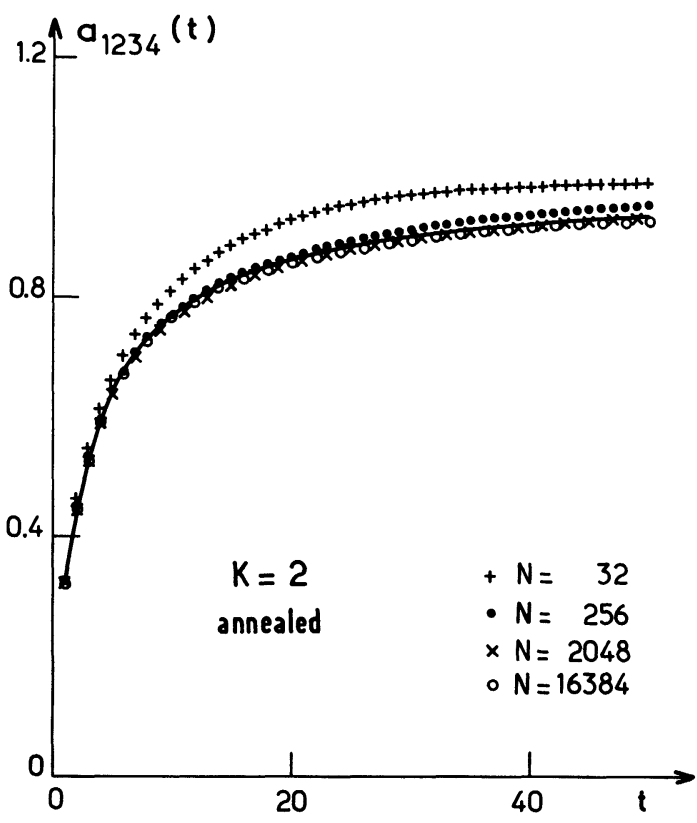

b)

Fig. 4. - Same conditions as in figure 2 for connectivity $K=2$ (overlaps between 4 configurations).

The log-log plot seems to indicate that $b_{12}$ and $b_{1234}$ converge to 1 as $N$ increases in the following way:

$$
\begin{aligned}
& 1-b_{12} \sim N^{-\alpha} \\
& 1-b_{1234} \sim N^{-\alpha}
\end{aligned}
$$

An estimate of the slope gives

$$
\alpha=0.27 \pm 0.03 \text {. }
$$

This figure 5 confirms the idea that the annealed and 
quenched models have the same time evolution of $a_{12}$ and $a_{1234}$ even at large time, provided that the size $N$ is large enough.

\section{Conclusion.}

We have seen that the time evolution of the overlaps of $3,4,5$ configurations can be computed for the annealed model and we have given the expressions of these time evolutions (Eqs. (7), (10), (12) and (4)).

Comparing the results of the numerical simulations, we have seen that for $N$ large enough, one cannot distinguish the time evolution of the quenched and of the annealed models. The following simple argument can explain this similarity.

The quenched model differs from the annealed model because of the correlations introduced by signals incoming on a given node following different paths. Let us consider a site $i$ at time $t$. It has $K$ input sites at time $t-1$. Each of these sites have $K$ input sites at time $t-2$ and so on up to initial time $t=1$. The value of $\sigma_{i}$ at time $t$ depends upon the functions $f_{j}$ located on $M$ sites.

$$
\begin{aligned}
M=1+K+K^{2}+ & \cdots+ \\
& +K^{t-1}=\left(K^{t}-1\right) /(K-1) .
\end{aligned}
$$

If these sites are all different, their are no correlations between functions $f_{j}$ and both models have identical behaviour. This occurs with a probability $P$

$$
P=\prod_{i=1}^{M}(1-i / N) .
$$

which, at finite time delays $t$, goes to 1 when $N$ goes to infinity. The two models thus have identical behaviours the thermodynamic limit.

Further refinements of the above argument are still needed in order to predict the correct time dependence of the finite size effects observed in our simulations.

\section{Acknowledgments.}

We would like to thank V. Hakim for an illuminating discussion and our referee for an interesting suggestion.

\section{References}

[1] Kauffman, S. A., J. Theor. Biol. 22 (1969) 437.

[2] Hopfield, J. J., Proc. Nat. Acad. Sci. USA 79 (1982) 2554.

[3] Kauffman, S. A. J. Theor. Biol. 44 (1974) 167.

[4] Kauffman, S. A., Physica D 10 (1984) 145, and other papers of the proceedings of Los Alamos Conference in this volume.

[5] Gelfand, A. E. and Walker, C. C., Ensemble Modelling (M. Dekker) 1984
[6] Fogelman Soulie, F., Goles Chacc, E. and Weisbuch, G., Bull. Math. Biol. 44 (1982) 715.

[7] Derrida, B. and Pomeau, Y., Europhys. Lett. 1 (1986) 45.

[8] Coste, J. and Henon, H., in Bienenstock, E., Fogelman Soulie, F., Weisbuch, G. Eds., Discordered Systems and Biological Organization. NATO ASI Series in Computer and Systems Sciences (Springer Verlag) 1986, p. 361. 